\title{
CORS ARCHITECTURE AND EVALUATION OF POSITIONING BY LOW-COST GNSS RECEIVER
}

\author{
Massimiliano PEPE \\ University of Naples "Parthenope", Italy \\ Received 13 April 2018; accepted 11 June 2018
}

\begin{abstract}
In recent years, the use of low cost GNSS receivers is becoming widespread due to their increasing performance in the spatial positioning, flexibility, ease of use and really interesting price. In addition, a recent technique of Global Navigation Satellite System (GNSS) survey, called Network Real Time Kinematic (NRTK), allows to obtain to rapid and accurate positioning measurements. The main feature of this approach is to use the raw measurements obtained and stored from a network of Continuously Operating Reference Stations (CORS) in order to generate more reliable error models that can mitigate the distance-dependent errors within the area covered by the CORS. Also, considering the huge potential of this GNSS positioning system, the purpose of this paper is to analyze and investigate the performance of the NTRK approach using a low cost GNSS receiver, in stop-and-go kinematic technique. By several case studies it was shown that, using a low cost RTK board for Arduino environment, a smartphone with open source application for Android and the availability of data correction from CORS service, a quick and accurate positioning can be obtained. Because the measures obtained in this way are quite noisy and, more in general, increasing with the baseline, by a simple and suitable statistic treatment, it was possible to increase the quality of the measure. In this way, this low cost architecture could be applied in many geomatics fields. In addition to presenting the main aspects of the NTRK infrastructure and a review of several types of correction, a general workflow in order to obtain quality data in NRTK mode, regardless of the type of GNSS receiver (multi constellations, single or many frequencies, etc.) is discussed.
\end{abstract}

Keywords: low-cost receivers, NRTK, GNSS, CORS, Arduino, DGPS.

\section{Introduction}

The availability of the Network Real Time Kinematic (NRTK) service allows the user to obtain a speed and accurate Global Navigation Satellite Systems (GNSS) positioning. In this environment, a key element is represented by Continuously Operating Reference Station (CORS) and the service for the positioning in real time (e.g. Rizos \& Satirapod, 2011; Lachapelle, Ryan, \& Rizos, 2002; Dobelis \& Zvirgzds, 2016). An increasing number of GNSS-CORS networks is being established and expanded around the world (e.g. Odijk \& Teunissen, 2011; Commins \& Janssen, 2012). Beyond the positioning system provided by institutional entities, such as national and local administrations, etc. there are several commercial NRTK services around the world (Aponte et al., 2009). For example, in Europe, North America, Australia and Russia, a service called SmartNet provides continuous and high level of accuracy to its subscribers (Leica Geosystems, 2017). Also, the spread of the positioning service by NRTK is of strategic importance and encouraging the use in different fields of geomatics applications, such as cultural heritage, monitoring civil engineering structure, tracking structures, cadastral surveys, mapping, land use monitoring etc. (e.g. Brutto, Garraffa, \& Meli, 2014; Djaja, Putera, Rohman, Nanditho, \& Suyanti, 2017; Pepe, 2017a). Indeed, the use of a NRTK network allows the user numerous advantages with respect to conventional RTK positioning: better coverage, greater reliability, more homogeneous accuracy, faster start up and higher profitability (Garrido, Giménez, Lacy, \& Gil, 2011).

In RTK environment, a common indicator used to test the quality of the point positioning, is the type of the solution: fixed and float. A fixed ambiguity is reached when the receiver has locked the carrier phase and calculated the integer value of the whole cycle counts from the receiver to each satellite for each frequency (Odolinski, 2010). This integer value is added to the partial cycle which the receiver records. The level of precision of the measuring is few centimetres and in this case the solution obtained is called

${ }^{*}$ Corresponding author. E-mail: massimiliano.pepe@uniparthenope.it 
"Fixed". The Float solution occurs when the receiver is not still able to fix the entire number of cycles to an integer. As a result, the accuracy obtainable is more than a few decimetres. Another type of correction method available by CORS infrastructure is the Differential Global Positioning Systems (DGPS) which calculates the position using a local DGPS base station. This method is based on the principle that all receivers in the same vicinity will simultaneously experience common errors (Hossam-e-Haider \& Qishan, 2000). Further, DGPS is applied in the code pseudo-rangers after estimating the corrections. This last type of correction can be obtained from some systems, such as Wide Area Augmentation System (WAAS) or European Geostationary Navigation Overlay Service (EGNOS) (Osório \& Cunha, 2013).

Using GNSS double frequency receivers and suitable antenna, the level of precision achieved is of few centimetres (Al-Shaery, Zhang, \& Rizos, 2013). However, testing precise positioning using NRTK corrections, limitations and potentiality are still under study and investigation, especially connected to the use of low cost receivers (Wiśniewski, Bruniecki, \& Moszyński, 2013). In order to demonstrate and verify the performance of a low cost RTK-GPS receiver, Takasu and Yasuda (2009) have developed a software called RTKLIB capable of interfacing with hardware components. The receiver achieved standard RTK-GPS accuracy of few centimetres while as regards the fixing ratio, values were of $50-60 \%$. Cai, Cheng, Meng, Tang, and Shi (2011) have developed and tested a prototype system using low-cost GPS receiver and the NRTK GPS. In this way, it was found that better than decimetre real-time on-the-fly positioning precision could be achieved. Dabove and Manzino (2014) discussed of the precision achievable using a low cost receiver; in this contexts the authors showed the level of precision was of few centimetres and the possible benefit of integration with other satellite constellations. Tsakiri, Sioulis, and Piniotis (2016) showed that the level of the accuracy is lower than $10 \mathrm{~cm}$ (3D positioning) and the "fixed" solutions were greater than $76 \%$; specifically, in an open environment, the level of precision achievable was few centimetres.

In this paper, after describing and deepening the NRTK architecture, as the framework network, the permanent stations, the data connection as a data exchange tool and positioning services, subsequently, the performance of low cost receiver was investigated. To achieve this goal, repeatability tests of the NRTK measures and range of precision were performed. In addition, the positions obtained by NRTK approach were compared with benchmarks belonging a regional network, in turn connected to the primary network. In this way, it was possible to obtain even an accuracy estimation.

\section{Architecture of the CORS infrastructure}

\subsection{Network geodetic frame}

Since its establishment in 1988, the International Earth Rotation Service (IERS) published on an annual basis a new realization of the International Terrestrial Reference System (ITRS). Each year, the IERS Central Bureau collected among contributing analysis centers their solutions for Earth Rotation Parameters together with the associated Terrestrial Reference Frame (TRF) data (Blewitt et al., 1998). These contributions are currently available by several space techniques such as Satellite Laser Ranging (SLR), Lunar Laser Ranging (LLR), Very Long Baseline Interferometry (VLBI), GPS and by the French Doppler system called DORIS (Kaplan \& Hegarty, 2005). Such a realization International Terrestrial Reference frame (ITRF) is now widely known under the label ITRFyy, where yy mean the reference year. The realization of this reference system is realized by a group of permanent stations (PS). To each PS is associated a set of three coordinates and three velocity parameters (with the variance/ covariance matrix) and for this reason, the ITRF defines a "dynamic" reference system. Also, the site coordinates refer to a precise reference epoch $t_{0}$. The position of a site, for a generic epoch $t \neq t_{0}$, can be computed by a linear propagation (Altamimi, Rebischung, Métivier, \& Collilieux, 2016):

$$
X(t)=X_{0}+\dot{X}\left(t-t_{0}\right)+\sum_{i} \Delta X_{i}(t),
$$

where: $X_{t}$ instantaneous position of a point on Earth Crust at epoch $t ; X_{0}$ point position at a reference epoch $t_{0} ; \dot{X}$ point linear velocity; $\Delta X_{i}(t)$ high frequency time variations: solid earth, ocean and pole tides, earthquakes.

Considering the variability dynamic aspect of each Permanent Station constituted an unnecessary complication for most applications. Therefore, in order to overcome this limitation, the territory was divided into an intraplate system of reference from the global reference system. For example, in Europe area, the European Terrestrial Reference System 1989 (ETRS89) constitutes the reference system for the Eurasian intra-plate (Gandolfi, 2015). The transition from ITRFyy to ETRFyy is developed by the EUREF (European Reference Frame) and can be realized by three following steps (Altamimi, Collilieux, Legrand, Garayt, \& Boucher, 2007; Caldera, 2010):

1. Coordinates estimation in the current ITRFyy, at the current epoch $(t)$, i.e. $\underline{X}_{\text {ITRF } 2005}(t)$.

2. Transformation from ITRF to ETRF, at the current epoch, using the Helmert transformation:

$$
\underline{X}_{E T R F}(t)=\underline{\operatorname{tr}}(t)+(1+k(t)) \Omega(t) \underline{X}_{\text {ITRF }}(t),
$$

where: $X_{E T R F}$ position vector in ETRF frame; Tr translation vector; $k$ scale factor; $\Omega$ rotation matrix; $X_{\text {ITRF }}$ position vector in ETRF frame.

$$
\left\{\begin{array}{l}
\underline{\operatorname{tr}}(t)=\underline{\operatorname{tr}}\left(t_{0}\right)+\underline{\dot{t}}\left(t-t_{0}\right) \\
k(t)=k\left(t_{0}\right)+\dot{k}\left(t-t_{0}\right) \\
\Omega(t)=\Omega\left(t_{0}\right)+\dot{\Omega}\left(t-t_{0}\right)
\end{array} .\right.
$$

Where all the terms reported in the formula (3) are tabulated in the official IERS catalogues available on the dedicate website. 
3. Propagation of the ETRF2000 coordinates obtained by formula (2), in the case of the ETRS89 coordinates are required in a time $\bar{t} \neq t$. In this case, the ETRF2000 coordinates can be computed by following formula:

$$
\underline{X}_{\text {ETRF } 2000}(\bar{t})=\underline{X}_{E T R F}(\bar{t})+(\bar{t}-t) \dot{X}_{E T R F 2000}(\bar{t}) \text {, }
$$

where $\dot{X}_{\text {ETRF2000 }}$ represent the ETRF2000 geodynamic velocities.

Therefore, the coordinates of each PS are continually updated. For example, on Italian territory, the I.G.M.I. (Italian Geographic Military Institute) have defined a new geodetic reference frame called RDN ("Rete Dinamica Nazionale" - "Dynamic National Network"). This network was framed in ETRF2000 and is characterized by 99 permanent GNSS stations (evenly distributed in the Italian territory) whose coordinates are calculated periodically to take account of natural changes in the crust surface (Barbarella, Gandolfi, Ricucci, \& Zanutta, 2009).

\subsection{The CORS services for user positioning}

The aim of NRTK for user positioning is to minimize the influence of the distance dependant errors on the computed position of a rover respect a specific network. Indeed, if the distance between the reference station is restricted to less than $40-50 \mathrm{~km}$, it is possible to obtain quick and reliable ambiguity resolution. In order to generate corrections for the network area, a minimum of three permanent stations is required. However, as the number of stations increases, redundancy increases and, as consequence, a better correction can be obtained. In addition, if one or two reference stations fail at the same time, their contribution can be eliminated from the solution and the remaining reference stations can still provide the user with corrections and give reliable results (El-Mowafy, 2012).

In principle, in accordance with El-Mowafy (2012) the NRTK approach consists of four basic segments:

- data collection at the Permanent Stations;

- manipulation of the data and generation of corrections at the network processing centre;

- broadcasting the corrections;

- positioning at the rover utilizing information from the NRTK.

The rover is to connect to the server by a one-way or double-way connection device (such as a radio modem, GSM or Internet). Once that the rover received the data, it calculates its position using the appropriate algorithm. Most common and different service available to users are available: Nearest station (NRT), Virtual Reference Station (VRS), Master Auxiliary (MAC or MAX or i-MAX) and Flächen-Korrektur-Parameter (FKP) (Cina, Dabove, Manzino, \& Piras, 2015).

A fast and easy positioning method of positioning in real time is the Nearest Differential Correction or Nearest station (NRT). In this method, the nearest permanent station to the user works as master station. Also, if the distance master-rover is below to $30 \mathrm{~km}$ and using suitable
GNSS receiver, NRT method allows to improve the user position by one PS belong the network. However, working with one station, the user losses the characteristic of reliability, accuracy, faster in start-up etc. than an multi station approach.

An approach multi-station widely spread is the "Virtual Reference Station". It is based on a network of GNSS reference stations continuously connected via data links to a control center; a computer at the control center continuously gathers the information from all receivers, and creates a living database of regional area corrections (Landau, Vollath, \& Chen, 2009). Also, the VRS requires the user to send the user's approximate position to the network control centre, which means that bidirectional communication between the user and the control centre is needed. The control centre then establishes a 'virtual' reference station close to this approximate position and calculates corrections for the virtual station. Indeed, the name of this approach results from the fact that observations for a "virtual" non-existing station are created from the real observation of a multiple reference station network (Retscher, 2002).

Another RTK positioning by CORS infrastructure is the FKP approach, which supports radio broadcasting and, as consequence, it is not needs for the rover to transmit its position. The server models distance-dependent errors and sends the RTK from a single reference within the network. The FKP creates area correction parameters with plain planes that are valid for a limited area around a single base (Usui et al., 2004).

Because the VRS and FKP approaches show some problems or limitations (Brown, Keenan, Richter, \& Troyer, 2005), it was developed another approach called MasterAuxiliary Concept (MAC). MAC correction is marketed in RTK products under the name of MAX and i-MAX. This approach is designed to transmit all relevant correction data from a CORS network to the rover in a highly compact form by representing ambiguity-levelled observation data as correction differences of dispersive and non-dispersive data for each satellite-receiver pair (Jansse, 2009). Indeed, in the MAC (or MAX, i-MAX) corrections, the server sends to the rover all observations and coordinates for a single reference station (master station) for all the other permanent stations, or for a subset of them, known as auxiliary stations; the observations are transmitted with ambiguity already reduced and the difference in coordinates. This difference it is related to the master station. So, this correction is a standard method because it uses public algorithms to generate corrections, it is traceable and repeatable because it is referring to real stations.

In general, the CORS infrastructure offers more types of corrections. However, it is important emphasize that while the MAC and FPK algorithm needs a double frequency GNSS receiver as rover, VRS and NRT can use also a single frequency receiver. For this reason, these latter corrections are widespread for applications that using low cost GNSS receiver. 


\subsection{Data correction format and GPRS transmission}

The transmission of differential corrections from the network server to rover can be obtained by different formats, such as CMR (Compact Measurement Record), CMR+, FKP (Flaechen-Korrektur-Parameter), FKP++ and RTCM (Radio Technical Commission for Maritime service). The difference among the formats is the amount of correction data that can be transmitted to a guidance device from an increasing number of satellite sources. While some of the formats mentioned above are specific to a manufacturer (for example CMR is format provide by Trimble company), the RTCM is independent. Therefore, RTCM is a standardized format universally recognized and sanctioned by the "RTCM recommended standards for differential GNSS".

A widespread technology for broadcast of the NRTK correction is the GPRS (General Packet Radio Service). It is an internet service provided by all mobile operators presents in the area where is present the GSM (Global System for Mobile Communications) signal. However, in general the signal broadcast generated from specific company does not cover all the territory; in other words, this mean that it is important to check if the survey area of interest is covered by GSM signal. This task can be obtained verifying presence of the signal by the website of the mobile phone company or visiting dedicated website that produce this information, such as "opensignalmaps". This latter website, available on worldwide scale, shows the different operators present in an area, the signal strength and location of antennas.

\section{Materials and method}

\subsection{Architecture of GNSS system developed for NRTK survey}

The prototype system developed in this experimentation consists of the following hardware components: external active GNSS antenna with SMA to u.FL cable assembly, RTK receiver module (GPS L1 + QZSS + SBAS C/A code), Bluetooth board, UARTtoUSB adapter, breadboard and Arduino jumper wires, Power Bank and a smartphone.

The RTK board, whose cost is less than $50 €$, is able to acquire data at $1 \mathrm{~Hz}$ update rate and can accept RTCM 3.x messages or other raw measurement data from a base station. In order to create the GNSS architecture, the RTK board was connected to Bluetooth board which, in turn, realized the connection with the Android smartphone. A SMA to u.FL cable allowed the connection between GNSS-RTK board and a specific type of GNSS antenna. The system was powered by external battery power bank (20000 mAh) connected to UARTtoUSB adapter board. In order to configure the RTK board in rover mode, a specific software supplied by the board manufacturer was used. In this way, it was possible to configure the correct firmware for RTK module in rover mode. As regards the software used in the experimentation phase, in order to obtain RTK correction data from the base (belonging to network permanent stations) to the rover using Internet mode, "NTRIP (Network Transport of RTCM data over IP) Client" provided by Lefebure.com company was used. In particular, using an Android smartphone and, as of consequence, the application called "NTRIP Client App", the NRTK data were acquired in this environment. The architecture configuration of low cost GNSS system developed is shown in the Figure 1.

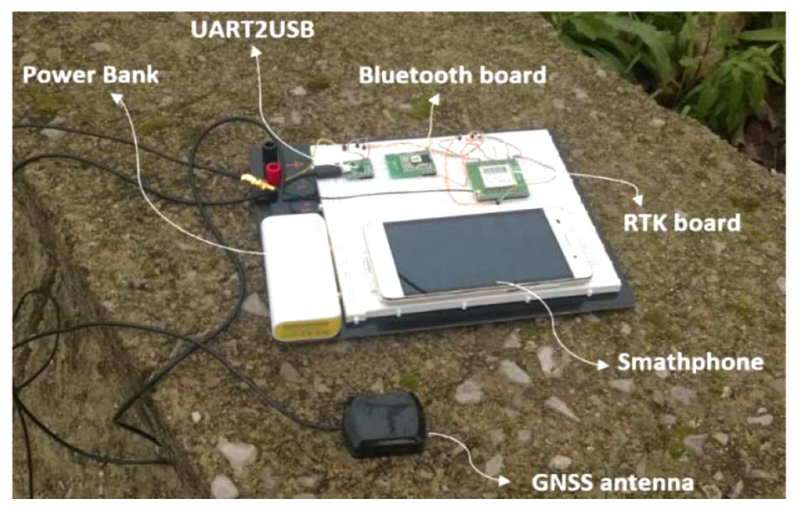

Figure 1. Architecture of the receiver developed

\subsection{Planning of NRTK survey}

Before starting each measurement session, in order to increase the probability of success of the GNSS survey in NRTK mode, it was necessary pay close attention to measuring planning. In this way, it was possible to reduce the error due a several sources, such as satellite geometry, number of satellites available, status of CORS, GSM signal availability etc.

The error sources due to poor satellite geometry can be analysed by satellite ephemeris (Kaplan \& Hegarty, 2005). Indeed, the GNSS almanac is a practical and convenient source to get the ephemeris for all the satellites in the constellation in order to know information about the "health" state of the entire satellite constellation and coarse data on every satellite's orbit. The almanacs are available to users every day from some websites, and in general, they are present in two formats: SEM (.al3) and YUMA (.alm).

The most common mode for evaluating the quality of positioning depending on a specific satellite constellation is the Position Dilution Of Precision (PDOP) parameter, which is representative of the relative geometric quality of the satellite constellation, and the number of satellites that can be tracked in a specific time of the day (e.g. HofmannWellenhof, Lichtenegger, \& Collins, 2012; Pepe, Fregonese, \& Scaioni, 2018). Conventional mission planning tools allow to identify the best and worst time periods for GPS data collection at any location thanks to the ability to import Digital Elevation Models (DEM) and ephemeris satellite configuration.

Recently, more websites that show the availability and the geometry of the satellite constellation, such as GNSS radar (http://www.taroz.net/GNSS-Radar. html), GNSS Mission Planning developed by Navmatix 
(http://gnssmissionplanning.com), GNSS PLANNING provided by Trimble company (http://www.trimble.com/ gnssplanningonline) allow to planning a GNSS survey.

However, to ensure the success of the NRTK survey, it is necessary to consider not only the parameter that influence directly the satellite constellation, but external parameters, such as the data transmission. In other words, mission planning in RTK GPS surveying must take into account even the visibility of the rover compared with NRTK infrastructure. In addition, the GNSS permanent network operator can provides a quality data analysis service and therefore, through a web service, it is possible to inform the users about the health of the network. An example of software developed according to the characteristics described above is the GNSS Spider Software Suite realized by Leica Geosystems, which is modular and scalable with new advanced solutions for long-range highaccuracy Network RTK, centralized data distribution and very useful tool in order to verify quality of the GNSS data.

Lastly, web-service provided by the mobile operator that shown phone signal coverage allows to verify the coverage and quality of the signal (as described previously in the 2.3 section). Also, taking in account the several components of the NTRK infrastructure, an example of workflow that should be adopted for NRTK survey, is sketched in Figure 2.

\subsection{Precision tests in open sky environment}

In order to verify the range of precision of NRTK measurement, repeatability tests were carried out. They consisted in the realization of more (real time) measurements on the same reference point. In particular, the measurements on the same test site were repeated for several days in order to acquire data in different satellite configurations and, as a consequence, to investigate the performance of the NRTK system in different scenarios. In each session, the maximum acceptable PDOP value for the acquisition data was 3. The algorithm used for the NRTK correction was "NeaRes T" and the mount point available from Italpos network was NRT3-RDN. Considering the density of the permanent station and because in general, the companies (or operators or public administration of the CORS) offer this service at a lower price than other corrections, it was decided to use this latter type of correction. Three test sites were individuated in relation to the distance from the master station, as shown in the Table 1 .

Table 1. Distance master-rover of test sites

\begin{tabular}{|c|c|}
\hline Test site & Baseline $(\mathrm{km})$ \\
\hline 1 & 0.543 \\
\hline 2 & 5.351 \\
\hline 3 & 19.293 \\
\hline
\end{tabular}

The ideal condition for NRTK survey was verified before every survey using the workflow developed and described in previous paragraph (see Figure 2). In particular, in order to know the status of the satellite configuration, the GNSS- RADAR web application (Kitamura, Yasuoka, \& Suzuki, 2013) was consulted. Alternatively, in any cases, Applanix PosPac software (developed by Trimble company) was used. As regards the status of the CORS, the Italpos website was consulted which by a simple and intuitive graphic representation, it showed a different colour the Permanent Station in relation to their status (green, means that the station is able to acquire and transmit data while red, means the station does not work). Lastly, as regards

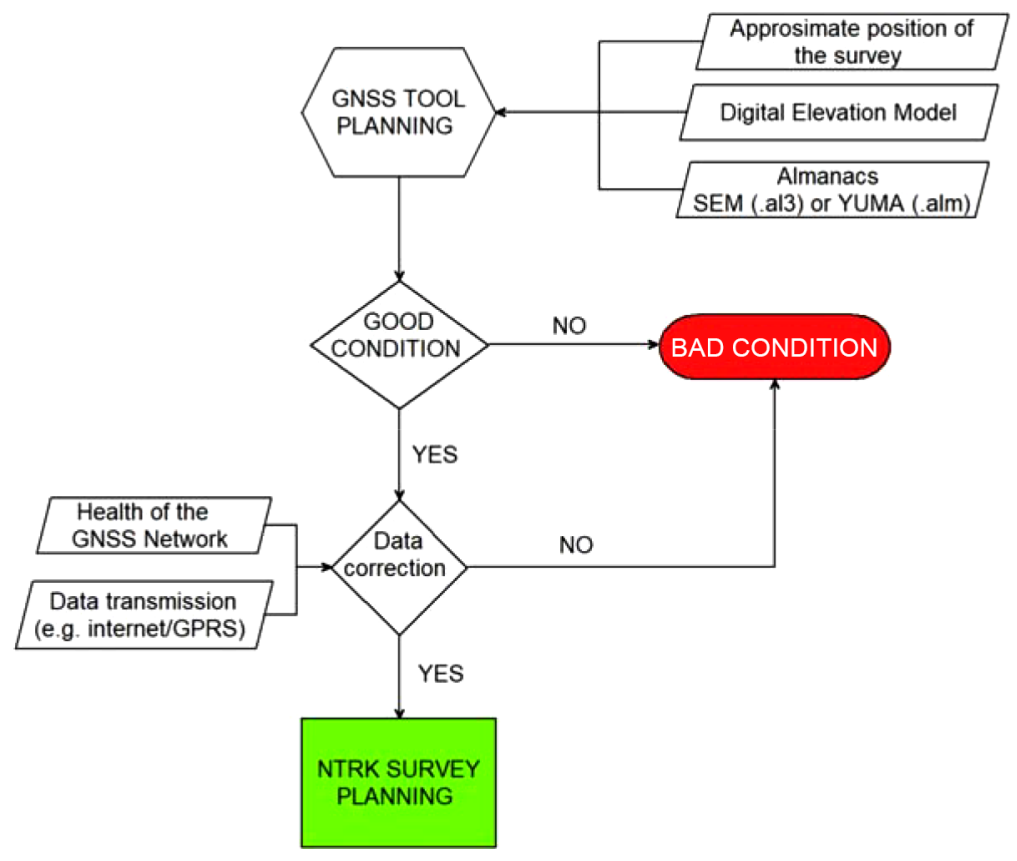

Figure 2. Workflow of NRTK survey planning 
the signal coverage, it was consulted the opensignalmaps website using TIM operator. At the end of the these several steps, it was verified if the conditions for the survey could be suitable.

Once performed this task, on each test site, eight NRTK measurements were carried out. In particular, on each test site, five measurement sessions were carried out. The results obtained are summarized in the following graphs (Figures 3, 4 and 5) generated by ArcGIS software (Version 10.4.1). In these graphs, the dots coloured represent the NRTK points acquired in the several sessions and each colour represents the position acquired in a different GNSS scenario. The reference system used for the experimentation was UTM33N-ETRF2000 projection. Because the software application provided the geodetic coordinates (longitude and latitude), in order to simplify the calculation operations, it was necessary to transform these coordinates into planar. This task was carried out by a specific routine developed in Matlab software.

The red triangle, showed in the Figures 3, 4 and 5, represents the reference point obtained by acquisition with dual frequency (L1, L2) GPS instrumentation (Hi Target V8 model) for a time of 30 minutes in static mode at the frequency of $1 \mathrm{hz}$. The calculation of the points in postprocessing mode (e.g. Parkinson \& Enge, 1996; Pepe, 2017b) was carried out using Hi-Target Geomatics Office

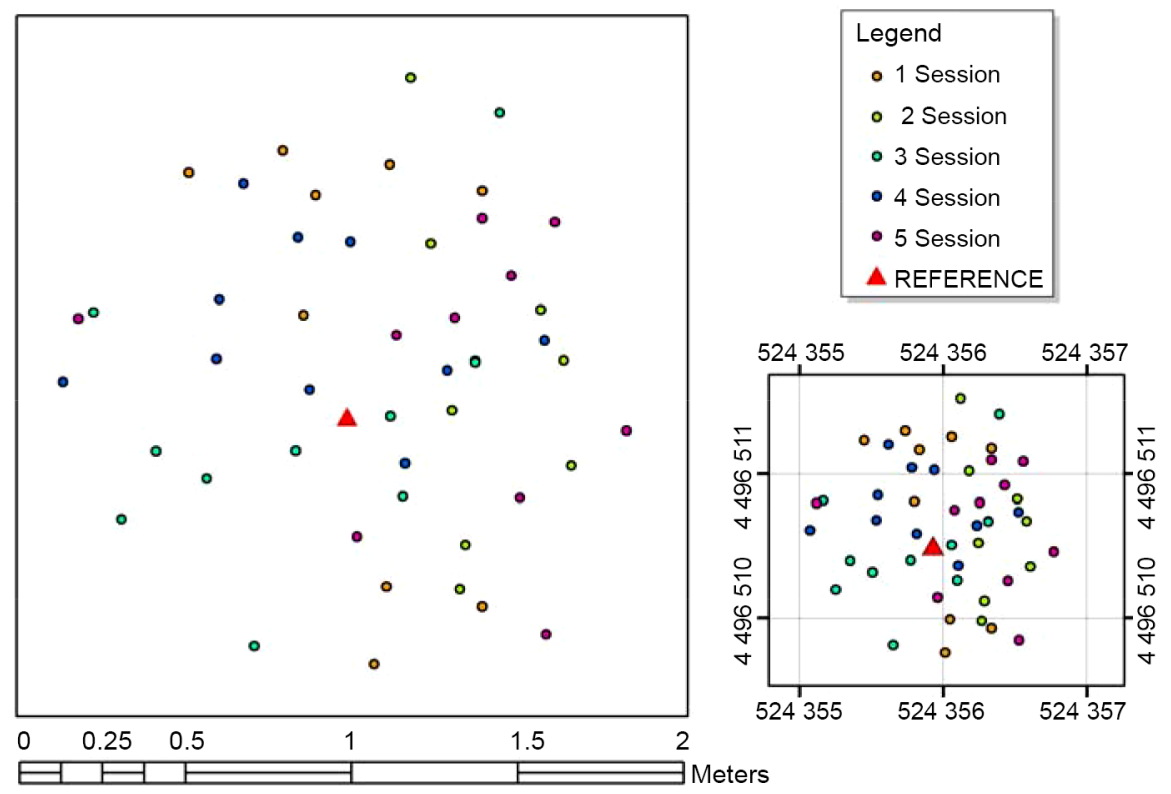

Figure 3. Spatial distribution of the points in different sessions on test site 1

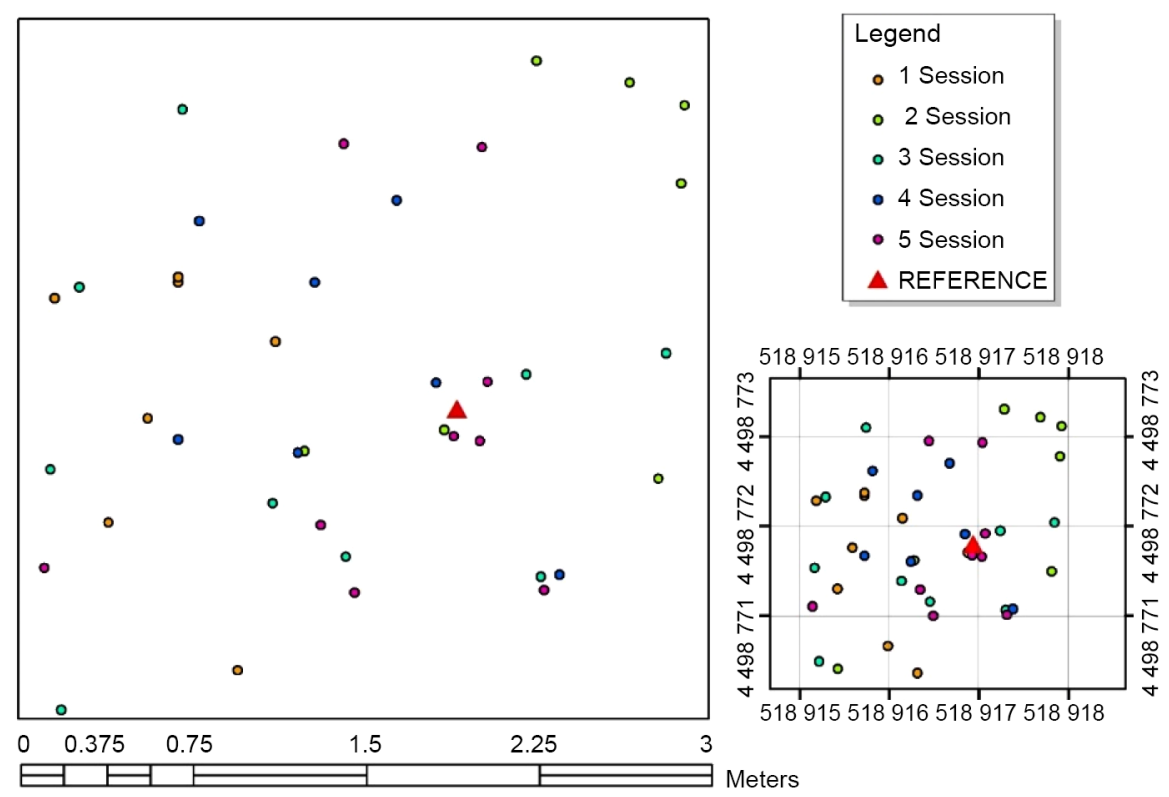

Figure 4. Spatial distribution of the points in different sessions on test site 2 


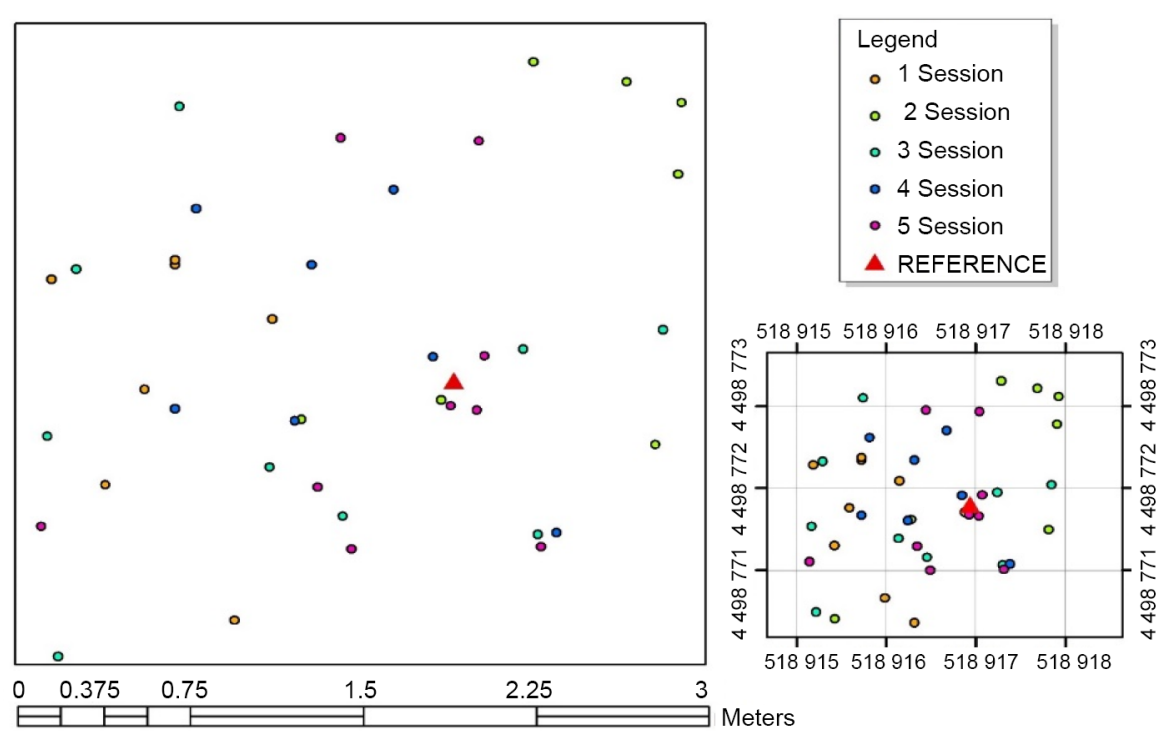

Figure 5. Spatial distribution of the points in different sessions on test site 3

(HGO) (Version 1.1.1) software and, as rinex files, were used those generated by the three nearest base station available from the network. In addition, the three stations were arranged in such a way as to represent the vertices of an equilateral triangle within which the point of observation is located. The elaborations steps used in order to calculate the coordinates of the tested points in static mode by HGO software, can be summarized as follows:

- Construction of the project:

- Import rinex from the CORS and raw data GPS instrument;

- Identification of the master and rover within the single project;

- Antenna height setting.

- Baseline Processing;

- Insert/edit the coordinates of the Permanent Stations;

- Setting of the parameters: tropospheric and ionospheric model, cut-off angle, fix ambiguities, frequency and sample rate.

- Network Adjustment.

At the of the processing, it was generated and exported the report of the static elaboration which showed a planimetric accuracy of $0.09 \mathrm{~m}$. Because GPS processing using the static method is very reliable, it was possible to compare the positioning obtained in this way that the positioning obtained in real time by low-cost instrument in the several sessions. Therefore, in the previous graphs (Figures 3, 4 and 5), beyond to visualizing how the points acquired in real time were spatial arranged, it was also possible to understand how much their positions differ from those generated by reliable tools and methods. In other words, in this way it was possible to carry out a qualitative analysis on the spatial distribution of the points with respect to a reference point.

\subsection{Evaluation of the accuracy on benchmark of known coordinates}

The accuracy test was conducted carrying out more measures on a reference vertex of known coordinates $\left(E_{R E F}\right.$, $\left.N_{R E F}\right)$. As reference points was chosen three vertices belong the Campania Region Network contained in few kilometres from master station. Because this network was realized in the ETRF89 frame, the vertexes were transformed in the recent ETRF2000 reference system using appropriate grid (provided by I.G.M.I.) containing the values for the system transition. In addition, thanks to development of algorithms in Matlab environment, it was possible to compute the coordinates of the vertices in the current projection system (Table 2).

Table 2. Coordinates of the check points

\begin{tabular}{|c|c|c|}
\hline \multirow{2}{*}{$\begin{array}{c}\text { Id Vertex } \\
\#\end{array}$} & \multicolumn{2}{|c|}{ Spatial coordinate (UTM33N-ETRF2000) } \\
\cline { 2 - 3 } & $E_{\text {REF }}(\mathrm{m})$ & $N_{\text {REF }}(\mathrm{m})$ \\
\hline 468376 & 524861.203 & 4498694.512 \\
\hline 468052 & 527102.993 & 4497223.482 \\
\hline 468031 & 526385.533 & 4496593.292 \\
\hline
\end{tabular}

On each vertex, more NRTK measurements were performed (15 measures). In this way, it was possible to calculate the average spatial plane coordinates derived by NRTK survey. In particular, using a normal distribution to only spatial dimension (i.e. for the single East and North coordinate) with a value of $2 \sigma$, it was possible to eliminate the potential outlier points. Subsequently, these values were compared with those of the reference point $\left(E_{R E F}\right.$, $N_{R E F}$ ) and the difference values achieved on the three check points, evaluated for the single spatial coordinate, are shown in the Table 3. 
Table 3. NRTK accuracy on three Check Points

\begin{tabular}{|c|c|c|c|}
\hline \multirow{2}{*}{$\begin{array}{c}\text { Id Vertex } \\
\#\end{array}$} & \multirow{2}{*}{$\begin{array}{c}\text { Baseline } \\
(\mathrm{km})\end{array}$} & \multicolumn{2}{|c|}{ Coordinate difference } \\
\cline { 3 - 4 } & & $\Delta N(\mathrm{~m})$ & $\Delta E(\mathrm{~m})$ \\
\hline 468376 & 2.1 & 0.343 & 0.996 \\
\hline 468052 & 3.2 & 1.109 & -0.603 \\
\hline 468031 & 2.5 & 0.669 & 0.704 \\
\hline
\end{tabular}

\section{Results and discussion}

In all test and check points used for the experimentation, the solution obtained was DGPS. Indeed, despite a long wait on one point, the solution did not change in better solutions. In fact, especially in the points where the distance base-rover was limited to few kilometres, the maximum error showed on the display of the smartphone was about $0.35 \mathrm{~m}$ and, this latter value, was represented the data acquisition threshold. In other words, when the error value (showed from the system in real time) was lower than $0.35 \mathrm{~m}$ than the point was acquired and stored. Instead, in the cases where the distance master-rover was greater than $3 \mathrm{~km}$ or the time acquisition in order to obtain an error lower than $0.35 \mathrm{~m}$ was very long, it was decided to acquire the spatial coordinates of the points when the accuracy achievable in real time was lower than $0.7 \mathrm{~m}$. However, in all places tested, the errors displayed in real time were lower than those actually verified, as shown even in the Figures 3, 4 and 5. Further, measurements sessions on the same points tested were carried using VRS correction and even in this case (i.e. using this last algorithm of correction), it was not possible to fix the ambiguities and the positioning on the tested check points did not make significant improvements compared to that obtained by Nearest correction.

Therefore, in order to verify the level of accuracy achievable by NRTK approach by Hi Target V8 GPS geodetic system in RTK mode (using Nearest correction) a series of measurements were realized. In other words, simultaneously with each survey performed with the lowcost system, a series of measurements with geodetic GPS was carried out. The maximum difference achievable between the points acquired with this geodetic instrument and regional benchmark (used as reference points), both in East and North coordinates, was $3.4 \mathrm{~cm}$. In addition, the system was always able to obtain fixed RTK solutions in few seconds. This means that if the project requires centimetres precisions in real time, the use of geodetics GNSS instruments is crucial.

\section{Conclusions}

In this paper it was shown, on one side the simplicity and positioning performance achievable by the use of low-cost GNSS receiver with differential correction obtained from the CORS, and on the other side the complex architecture necessary for their realization, continuous update of the coordinates of the single permanent stations and software management of NRTK infrastructure.

The use of low-cost GNSS receiver connected to CORS service allowed to obtain even sub-metric precision if the range from (permanent station) base-rover is limited to few kilometres and after performed a suitable statistical treatment on the acquired data. Therefore, considering the level of accuracy achievable by low-cost GNSS receivers (able to receive the correction from the CORS) can be used in specifics applications in the geomatics fields.

\section{Conflicts of interest}

The author declare no conflict of interest.

\section{References}

Al-Shaery, A., Zhang, S., \& Rizos, C. (2013). An enhanced calibration method of GLONASS inter-channel bias for GNSS RTK. GPS solutions, 17(2), 165-173. https://doi.org/10.1007/s10291-012-0269-5

Altamimi, Z., Collilieux, X., Legrand, J., Garayt, B., \& Boucher, C. (2007). ITRF2005: A new release of the International Terrestrial Reference Frame based on time series of station positions and Earth Orientation Parameters. Journal of Geophysical Research: Solid Earth, 112(B9). https://doi.org/10.1029/2007JB004949

Altamimi, Z., Rebischung, P., Métivier, L., \& Collilieux, X. (2016). ITRF2014: A new release of the International Terrestrial Reference Frame modeling nonlinear station motions. Journal of Geophysical Research: Solid Earth, 121(8), 6109-6131. https://doi.org/10.1002/2016JB013098

Aponte, J., Meng, X., Hill, C., Moore, T., Burbidge, M., \& Dodson, A. (2009). Quality assessment of a network-based RTK GPS service in the UK. Journal of Applied Geodesy, 3(1), 2534. https://doi.org/10.1515/JAG.2009.003

Barbarella, M., Gandolfi, S., Ricucci, L., \& Zanutta, A. (2009). The new Italian geodetic reference network (RDN): a comparison of solutions using different software packages. In Proceedings of EUREF Symposium (pp. 27-30). Florence, Italy,

Blewitt, G., Boucher, C., Davies, P. B. H., Heflin, M. B., Herring, T. A., \& Kouba, J. (1998). ITRF Densification and continuous realization by the IGS. In Advances in positioning and reference frames (pp. 8-17). Springer, Berlin, Heidelberg. https://doi.org/10.1007/978-3-662-03714-0_2

Brown, N., Keenan, R., Richter, B., \& Troyer, L. (2005, September). Advances in ambiguity resolution for RTK applications using the new RTCM V3.0 Master-Auxiliary messages. In Proc of ION GNSS 2005, Long Beach, California.

Brutto, M. L., Garraffa, A., \& Meli, P. (2014). UAV platforms for cultural heritage survey: first results. ISPRS Annals of the Photogrammetry. Remote Sensing and Spatial Information Sciences, 2(5), 227. https://doi.org/10.5194/isprsannals-II-5-227-2014

Cai, Y., Cheng, P., Meng, X., Tang, W., \& Shi, C. (2011). Using network RTK corrections and low-cost GPS receiver for precise mass market positioning and navigation applications. In Intelligent Vehicles Symposium (IV), pp. 345-349. https://doi.org/10.1109/IVS.2011.5940570

Caldera, S. (2010). GNSS permanent networks monitoring: problems and solution: $\mathrm{PhD}$ Thesis. 
Cina, A., Dabove, P., Manzino, A., \& Piras, M. (2015). Network Real Time Kinematic (NRTK) positioning-description, architectures and performances. https://doi.org/10.5772/59083

Commins, R., \& Janssen, V. (2012). Improving GNSS CORS Design: The CORSnet-NSW AdjusTable Antenna Mount (CAAM). Journal of Global Positioning Systems, 11(2), 109115. https://doi.org/10.5081/jgps.11.2.109

Dabove, P., \& Manzino, A., M. (2014). GPS \& GLONASS massmarket receivers: positioning performances and peculiarities. Sensors, 14(2), 22159-22179.

https://doi.org/10.3390/s141222159

Djaja, K., Putera, R., Rohman, A. F., Nanditho, I. S. G., \& Suyanti, E. (2017). The integration of geography information system (GIS) and global navigation satellite system-real time kinematic (GNSS-RTK) for land use monitoring. International Journal of GEOMATE, 13(36), 31-34.

https://doi.org/10.21660/2017.36.2768

Dobelis, D., \& Zvirgzds, J. (2016). Network RTK performance analysis: a case study in Latvia. Geodesy and Cartography, 42(3), 69-74.

https://doi.org/10.3846/20296991.2016.1226383

El-Mowafy, A. (2012). Precise real-time positioning using Network RTK. In Sh. Jin (Ed.), Global navigation satellite systems: signal, theory and applications (7, pp. 161-188). InTech. https://doi.org/10.5772/29502

Gandolfi, S. (2015), Impact of the updating of the National geodetic reference frame. iBollettino dell'Associazione Italiana di Cartografia, 153, 51-62.

Garrido, M. S., Giménez, E., de Lacy, M. C., \& Gil, A. J. (2011). Testing precise positioning using RTK and NRTK corrections provided by MAC and VRS approaches in SE Spain. Journal of Spatial Science, 56(2), 169-184.

https://doi.org/10.1080/14498596.2011.623341

Hofmann-Wellenhof, B., Lichtenegger, H., \& Collins, J. (2012). Global positioning system: theory and practice. Springer Science \& Business Media.

https://doi.org/10.1007/978-3-7091-6199-9

Hossam-e-Haider, M., \& Qishan, Z. (2000). Comparative study on OEM-based differential GPS. In Proceedings of the $13^{\text {th }}$ International Technical Meeting of the Satellite Division of the Institute of Navigation (pp. 305-310).

Janssen, V. (2009, December). A comparison of the VRS and MAC principles for network RTK. In IGNSS 2009 Symposium. Surfers Paradise, Australia.

Kaplan, E., \& Hegarty, C. (2005). Understanding GPS: principles and applications. Artech house.

Kitamura, M., Yasuoka, Y., \& Suzuki, T. (2013). Path planning for autonomous vehicles using QZSS and satellite visibility map. Journal of Robotics and Mechatronics, 25(2), 400-407. https://doi.org/10.20965/jrm.2013.p0400

Lachapelle, G., Ryan, S., \& Rizos, C. (2002). Servicing the GPS user. In J. Bossler, J. Jenson, R. McMaster \& C. Rizos (Eds.), Manual of geospatial science and technology (Chapter 14, pp. 201-215). Taylor \& Francis Inc.

Landau, H., Vollath, U., \& Chen, X. (2009). Virtual reference station systems, Positioning, 1(2).

Leica Geosystems. (2017). Last accessed 18/02/2017. Retrieved from http://leica-geosystems.com/services-and-support/ smartnet---satellite-positioning
Odijk, D., \& Teunissen, P. (2011). A theoretical study on the bottlenecks of GPS phase ambiguity resolution in a CORS RTK Network. Journal of Geodetic Science, 1(2), 143-153.

https://doi.org/10.2478/v10156-010-0017-0

Odolinski, R. (2010). Swedish user guidelines for network RTK. In Proceedings of the XXIV FIG International Congress. Sydney, Australia.

Osório, I., \& Cunha, M. (2013). The role of the TRS in precision agriculture: DGPS with EGNOS and RTK Positioning using data from NTRIP streams. In Reference frames for applications in geosciences (pp. 277-282). Berlin, Heidelberg: Springer. https://doi.org/10.1007/978-3-642-32998-2_40

Parkinson, B. W., \& Enge, P. K. (1996). Differential GPS. In B. W. Parkinson, J. J. Spilker, P. Axelrad \& P. Enge (Eds.). Global Positioning System: Theory and Applications (Vol. 2, pp. 3-50). Washington: American Institute of Aeronautics and Astronautics.

https://doi.org/10.2514/5.9781600866395.0003.0050

Pepe, M. (2017a). Use of digital aerial photogrammetry sensors for land cover classification. International Journal of Applied Engineering Research, 12(24), 15610-15620.

Pepe, M. (2017b). A survey by Airborne Laser Scanner of open large structure: A case study of Pompeii Amphitheatre. ARPN Journal of Engineering and Applied Sciences, 12(21), 1-11.

Pepe, M., Fregonese, L., \& Scaioni, M. (2018). Planning airborne photogrammetry and remote-sensing missions with modern platforms and sensors. European Journal of Remote Sensing, 51(1), 412-435.

https://doi.org/10.1080/22797254.2018.1444945

Retscher, G. (2002). Accuracy performance of Virtual Reference Station (VRS) networks. Positioning, 1(3).

https://doi.org/10.5081/jgps.1.1.40

Rizos, C., \& Satirapod, C. (2011). Contribution of GNSS CORS infrastructure to the mission of modern geodesy and status of GNSS CORS in Thailand. Engineering Journal, 11(1), 25-42.

https://doi.org/10.4186/ej.2011.15.1.25

Takasu, T., \& Yasuda, A. (2009). Development of the low-cost RTK-GPS receiver with an open source program package RTKLIB. In International Symposium on GPS/GNSS (pp. 4-6). Jeju, Korea: International Convention Centre.

Tsakiri, M., Sioulis, A., \& Piniotis, G. (2016). The use of low-cost, single-frequency GNSS receivers in mapping surveys. Survey Review, pp 1-11.

https://doi.org/10.1080/00396265.2016.1222344

Usui, S., Higuchi, H., Kanda, J., Wakimoto, K., Tanaka, S., \& Satoh, F. (2004, June). Nation-wide RTK-GPS based on FKP method and applications for human navigation and location based services. In 2004 IEEE International Conference on Multimedia and Expo (ICME) (vol. 3, pp. 1587-1590). Taipei, Taiwan.

https://doi.org/10.1109/ICME.2004.1394552

Wiśniewski, B., Bruniecki, K., \& Moszyński, M. (2013). Evaluation of RTKLIB's Positioning Accuracy Using low-cost GNSS Receiver and ASG-EUPOS. TransNav: International Journal on Marine Navigation and Safety of Sea Transportation, 7(1), 79-85.

https://doi.org/10.12716/1001.07.01.10 